\title{
Evaluación Nutricional en Lactantes Hospitalizados
}

\author{
Dr. Mario Becerra O. 1; Dra. Silvia Ibáñez T.1; Dr. Carlos Castillo D. 1,2 \\ Nutritional Evaluation of Inpatients at a Children's Hospital
}

\begin{abstract}
This work was done to assess the possible effects of prolonged hospital admissions on the nutritional status of infants. Infants and children 1 to 24 months old with ten days long minimal hospital admissions were weekly evaluated, up to thitty days in hospital, from July 1983 to January 1984: weight, height, upper arm circumference (AC), tricipital skinfold (TS), plasna albumin and hemoglobin were measurcd. Hospital stay penods were longer in infants with weight-for-age (W/A) or weight-for-height (W/H) rates less than 90\% of NCHS standards compared with normals ( $W / A$ and $W / H 100 \%)(31 \pm 28$ vs $18 \pm 9$ days, $<<0.05)$. Low respiratory tract infections were the major causes of admission to infants's wart's but diartheal disease was most prevalent anong patient with W/A $\leqslant 80 \%$ Children with AC below $S$ th percentile of standards were more frequently found among patients with longer stays and $\mathrm{AC}<5$ th percentle were well related to W/A $\leqslant 80 \%$ of standards. Hypoalbuminemia was unfrequent $(<16 \%$ but blood hemoglobin concentration less than 11 or $10,5 \mathrm{~g} / \mathrm{dl}$ was most prevalent $(>70 \%)$.

(Key words: Nutrition. Hospital permanence. Prolonged. Weight for age. Weight for stature. Braquial perimeter. Tricepts skinfold).
\end{abstract}

La preocupación por la nutrición de los niños se ha expresado generalmente en la atención ambulatoria. Los pacientes hospitalizados han recibido una atención muy secundaria en este aspecto, debido principalmente a que el deterioro tutricional que sufren se atribuía principalmente a sus enfermedades de base, considerándolo, por lo tanto poco susceptible de mejorar mientras esta no fuese controlada.

En los últimos años, gracias a distintos estudios, este concepto ha sido modificado a medida que se ha comprendido mejor la interrelación entre nutrición, infección y otros procesos mórbidos $^{1}$. Hoy se considera posible, en la mayoria Je los casos, mejorar la evolución de determinadas enfermedades con apoyo nutricional adecuado ${ }^{2}$.

Simultáneamente, han aunientado los pará. metros disponibles para evaluación nutricional, agregándose, a los procedimientos clásicos (peso y talla) mediciones de pliegues cutáneos, perímetro braquial, y, otros de laboratorio como albuminemia, creatininuria/talla, transferrina, proteina ligante de retinol, etc. cuyo fin es valorar con más exactitud el deterioro nutricional y sus repercusiones en la masa magra, la sintesis proteica y la inmunidad $3,4,5,6$.

1. Servicio de Pediatría, Hospital Sótero del Río, Santiago.

2. Instituto de Nutrición y Tecnología đe los Alimentos (INTA). Universidad de Chile.
Este trabajo se realizó con el propósito de determinar si existe deterioro en la nutrición en lactantes cuyo período de hospitalización excede la duración promedio habitual y si el empleo simultáneo de diferentes parámetros mejora la sensibilidad de los métodos para detectarlo.

\section{MATERIA L Y METODOS}

Se realizó un estudio prospectivo en los meses de Julio 1983 a Enero de 1984. En este período se evaluaron todos los lactantes (pacientes de 1 mes a 2 años de edad) que cumplieron 10 dias de hospitalización, plazo que se escogió por ser el promedio de hospitalización en nuestrd unidad de lactantes y por estitnarse que aquellos cuya estadía dura más que este plazo tendrían enfermedades más severas y mayor riesgo de deterioro nutricional int tahospitalario.

La muestra inciuyó a 228 lactantes, $41,8 \%$ de los pacientes ingresados en ese rango de edades, 134 eran hombres y 94 mujeres; su edad promedio al ingreso fue $6,6 \pm 4,5$ D.E. meses. El peso de nacimiento promedio fue de $2,9 \pm 0,8$ D.E. kg y el promedio de días de estada $24,5 \pm 22,7$ D.F. días.

Diariamente se visitó toda la Unidad de Lactantes ingresando de inmediato al estudio los pacientes que hubieran cumplido 10 días de hospitalización. Los casos que completaron dicho tiempo durante los fines de semana fueron incorporados el día lunes inmediatamente siguiente.

La información del ingreso se obtuvo del registro clínico, incluyendo peso y talla, como también las correspondientes al momento de cada evaluación. En todos los sujetos en que ello fue posible se midieron ađemás, pliegues tricipitales (PT) con calibrador tipo Langue de pliegues, perímetros braquiales (PB) con 
cinta metálica y se tomaron muestras de sangre para hemoglobina y concentración plasmática de albúmina.

Los pacientes fueron evaluados cada 7 días, contando desde el $10^{\circ}$ de hospitalización, hasta el alta o hasta cumplir 31 días en el hospital. Se determinaron las retaciones peso/edad (P/E) y peso/talla (P/T) de acuerdo al porcentaje de los estándares del "National Center for Health Statistics" (NCHS), aproximando la edad al mes mús cercano ${ }^{7}$. El plicgue tricipital (PTr) y el perimetro braquira (PB) fueton evaluados según las tablas de Frisancho, considerando como límite normal cl percentil $5^{\circ}$. Se consideraron anormales todas albumine. mias inferiores a $\mathbf{3 , 5} \mathrm{g} . \%$ y las concentraciones de hemoglobina inferiores a $10,5 \mathrm{~g} . \%$ en lactantes de 1 a 5 meses, y a $11 \mathrm{~g} . \%$ en los de 6 a 24 meses de edad. Ẽn el décimo día se evaluaron $22 \lambda$ pacientes, en el décinoseptimo 105 , a los 24 días 61 y en el día $31^{\circ} 34$ lactantes según $\mathrm{P} / \mathrm{l}$ : y $\mathrm{P} / \mathrm{T}$.

\section{RESULTADOS}

El porcentaje de lactantes con compromiso nutricional expresado como $\mathrm{P} / \mathrm{E}$ y $\mathbf{P} / \mathrm{T} \leqslant 90 \%$ en los distintos días de estudio, aparece en la Tabla 1. El día 0 corresponde al dia del ingreso al hospital de los pacientes incluidos en el estudio. El denominador no corresponde al total de niños evaluados, debido a que la información sobre peso y talla no siempre había sido consignada en la ficha clínica. Tambien fue variable el número de casos, en quienes se registraron los parámetros bioquímicos y se hicieron mediciones de pliegue tricipital y perimetro braquial.

Las principales causas de hospitalización fueron las enfermedades respiratorias bajas $(49,1 \%)$, y el síndrome de diarrea aguda con deshidratación $(32,5 \%)$.

La duración de la hospitalización en relación con el estado nutricional al ingreso, fue significativamente mayor en los pacientes cuyo $\mathrm{P} / \mathrm{E}$ era
Tabla 1.

Prevalencia de Desnutrición según Relaciones Peso/Edad (P/E), Peso/Talla (P/T), y Períodos ел Estudio en Lactantes Hospitalizados por más de 10 días.

\begin{tabular}{|c|c|c|c|c|}
\hline \multirow[b]{2}{*}{ Día } & \multicolumn{2}{|c|}{$P / E \leqslant 90 \%$} & \multicolumn{2}{|c|}{$P / T \leqslant 90 \% *$} \\
\hline & $\mathrm{N}^{\circ}$ & $\%$ & $\mathrm{~N}^{0}$ & $\%$ \\
\hline 0 & $128 / 213$ & 60.11 & $73 / 169$ & 43.2 \\
\hline 10 & $137 / 221$ & 62.0 & $77 i_{183}$ & 42.1 \\
\hline 17 & $79 / 105$ & 75.2 & $45 / 77$ & 58.4 \\
\hline 24 & $54 / 61$ & 88.5 & $22 / 44$ & 500 \\
\hline 31 & $27 / 34$ & 79.4 & $13 / 28$ & 46.4 \\
\hline
\end{tabular}

* * de los estándares OMS 1979.

$\leqslant 90 \%$ que en los eutróficos ( $18 \pm 9$ dias $v / s 31 \pm$ 28 días $\mathrm{p}<0.001$ ). No se obseryaron diferencias estadisticamente significativas en este aspecto al comparar criterios peso/edad y peso/talla.

En la Tabla 2, se ordenan los diagnósticos de ingreso de acuerdo al estado nutricional evaluado por la relación peso/edad. Las diarreas agudas pasan a constituir la primera caus de hospitalización en pacientes con $\mathrm{P} / \mathrm{E} \leqslant 80 \%$ y los pacientes con enfermedades crónicas concomitantes se acumulan en este mismo grupo. Las afecciones crónicas consistían principalmente en cardiopatias congénitas ${ }^{5}$, trisomia $21^{3}$, neumopatía crónica ${ }^{3}$. datio neurológico ${ }^{4}$, hiperplasia suprarenall.

El perímetro braquial fue evaluado en 190 pacientes a los 10 días; en 80 a los 17 días; en 47

Tabla 2.

Estado de la Nutrición según Relación Peso/Edad (P/E) en Lactantes Hospitalizados por más de 10 días, según Diagnóstico de Ingreso.

\begin{tabular}{|c|c|c|c|c|c|c|}
\hline \multirow{2}{*}{ Diagnóstico de Ingreso } & \multicolumn{2}{|c|}{$\mathrm{P} / \mathrm{E}>90 \% * *$} & \multicolumn{2}{|c|}{$90 \%+$ PE $80 \%$} & \multicolumn{2}{|c|}{$\mathrm{PE}+80 \%$} \\
\hline & $n$ & $\%$ & $\mathrm{n}$ & $\%$ & $\bar{n}$ & $\%$ \\
\hline Enf. Respiratoria & 58 & 67,4 & 21 & 50,0 & 32 & 38,5 \\
\hline Diarrca aguda & 14 & $16,3^{*}$ & 16 & 38,1 & 39 & $47,0^{\mathrm{*}}$ \\
\hline Otras afecciones agudas & 14 & 16,3 & 5 & 11,9 & 12 & 14.5 \\
\hline $\begin{array}{l}\text { Enfermedades crónicas } \\
\text { concomitantes }\end{array}$ & 3 & & 2 & & 12 & \\
\hline TOTAL & 86 & 100 & 42 & 100 & 83 & .100 \\
\hline
\end{tabular}


a los 24 dias y en 36 a los 31 días y resultó bajo el percentil 5 en $42,1 \%, 56,2 \%, 61,7 \%$ y $47,2 \%$ de ellos respectivamente, con lo que se observa una tendencia creciente de pacientes con $\mathrm{PB}$ bajo el percentil 5 al prolongarse la hospitalización hasta los 24 días.

La Tabla 3 muestra fa relación entre $\mathrm{PB}$ bajo el percentil 5 y el estado nutricional expresado como peso/edad. A pesar que los estándares de Frisancho no están totalmentc probados para lactantes, ni tampoco para nuestra población infantil, se encontró una relación bastante adecuada entre aquellos con PB bajo el percentil $5 y$ la relación $\mathrm{P} / \mathrm{E} \leqslant 80 \%$. No ocurre lo mismo al relacionar el $\mathrm{PB}$ con la relación $\mathrm{P} / \mathrm{T}$, observátidose con esta última aproximadamente un $35 \%$ de los pacientes en cada uno de los grupos estudiados $>90 \%, 90 \%$ a $80 \%$, $\leqslant 80 \%$ ).

Tabla 3.

Relación entre Núnero de Lactantes con PB < Percentil 5 y Estado Nutritivo Medido por Peso/Edad, en dos momentos del Estudio.

\begin{tabular}{|c|c|c|c|c|}
\hline \multirow{3}{*}{$\mathrm{P} / \mathrm{E}$} & \multicolumn{4}{|c|}{ Perímetro Braquial $<$ ps } \\
\hline & \multicolumn{2}{|c|}{10 días } & \multicolumn{2}{|c|}{24 días } \\
\hline & II & $\overline{\%}$ & $n$ & $\%$ \\
\hline$>90 \%$ & 13 & 16,3 & 1 & 3.4 \\
\hline$\leqslant 90 y>80 \%$ & 10 & 12,5 & 4 & 13,8 \\
\hline$\leqslant 90 \%$ & 57 & 71.2 & 24 & 82,8 \\
\hline TOTAL & 80 & 100 & 29 & 100 \\
\hline
\end{tabular}

El pliegue tricipal (PTr) fue evaluado sólo en los pacientes cuyo PB era inferior al percentil 5 (P5): Si P/E $\leqslant 80 \% 51 \%$ de los pacientes tenían $\mathrm{PTr}<\mathrm{P} 5$, cuando $\mathrm{PE} \leqslant 90 \%>80 \% \mathrm{PTr}$ era $<\mathrm{P} 5$ en 30\% y si PE era $>90 \%$ se encontraron $\mathrm{PT}_{\mathrm{r}}<$ Ps sólo en 15,3\% de los casos.

La hipoalbuminemia fue menos frecuente en los controles semanales posteriores a los 10 días. La proporción de pacientes con $\mathrm{Hb}$ đisminuida mostró tendencia a aumentar en las hospitalizaciones más prolongadas, pero este hecho no guardó relación con el estado nutricional medido por peso/edad o por peso/talla (Tabla 4).

\section{DISCUSION}

El estudio más a fondo de parámetros de riesgo nutricional en pacientes hospitalizados, ha cobrado importancia en los últimos años, a partir de estudios efectuados principalmente en adultos. Blackbourn y Bistrian ${ }^{9}$ demostraron en 1977 una asociación entre el deterioro de algunos parámetros antropométricos y bioquímicos, la
Tabla 4.

Evaluación Nutricional por Parámetros Bioquimicos en Lactantes Hospitalizado? por más de 10 días.

\begin{tabular}{lrrrrr}
\hline \multirow{2}{*}{ Día } & \multicolumn{2}{c}{ Albúmina* } & & \multicolumn{2}{c}{ Hemoglobina** } \\
\cline { 2 - 5 } & \multicolumn{2}{c}{ No $^{\circ}$} & $\%$ & & \multicolumn{2}{c}{ No $^{\text {\% }}$} & $\%$ \\
\hline 10 & $29 / 185$ & 15.7 & & $97 / 132$ & 73.5 \\
$1 ?$ & $22 / 144$ & 15.3 & & $83 / 110$ & 75.5 \\
24 & $8 / 86$ & 9.3 & $47 / 58$ & 81.0 \\
31 & $2 / 38$ & 5.2 & $19 / 21$ & 90.5 \\
\hline
\end{tabular}

* Albuminemia $<3,5 \mathrm{~g} \times \mathrm{dl}$

** Hemoglobinemia $<$ i0,5 $\mathrm{x}$ d $<$ reses $y$

$11 \mathrm{~g} . \%$ en niños con $<6$ neses, $d c$ edad.

prolongación de la permanencia en el hospital y complicaciones infecciosas y quirúrgicas. Un buen apoyo nutricional podría ser capaz de reducir el impacto de estas compliaciones. Varios estudios posteriores en adultos, han comprobado estos hallazgos y contribuido a adaptar la evaluación nutricional y las medidas de apoyo a cada problema en particular.

En Chile, se han publicado pocos estudios sobre el impacto nutricional que ocurre en niños hospitalizados ${ }^{10}$ : uno transversal, realizado en unn hospital pediátrico, demostró alta incidencia de deterioro y asociación con dietas insuficientes ${ }^{11,12}$. Otro demostró que un número significativo de lactantes desnutridos severos había adquirido esa condición después de haber estado hospitalizados $\$ 3$.

Para realizar una vigilancia nutricional efectiva es necesario emplear un sistema sencillo, de fáci] aplicación, cuyos parámetros tengan alguna capacidad de predicción del compromiso nutricional posterior $y$ las complicaciones médicas $e$ infecciosas de la enfermedad de base.

No es extraño que la diarrea sea la principal causa de hospitalización en desnutridos, pues en los pacientes afectados tiener mayor importancia las malas condiciones higiénicas y socioeconómi. cas, que también influyen en el estado de nutrición, que es agravado por la reducción de la ingestión de alimentos, las pérdidas intestinales de nutrientes y las demandas originadas por la fiebre.

La proporción de desnutridos entre los pacientes hospitalizados en esta serie fue significativamente mayor que el que existía en la población general; lo que también ha sido observado en otros estudios nacionales 11,14 . El deterioro observado en la relación $\mathrm{P} / \mathrm{E}$ al prolongarse la 
hospitalización, no ocurrió con la relación $\mathrm{P} / \mathrm{T}$, lo que podría ser atribuido a una detención precoz del incremento de la talla. En todo caso en este trabajo, no es posible descartar defectos en et registro de la talla, pues fue hecho por un gran número de personas distintas.

El perímetro braquial mostró buena corre. lación con el estado de nutrición medido por $\mathrm{P} / \mathrm{E}$, no así con $\mathrm{P} / \mathrm{T}$. Este hecho es importante porque este parámetro es sencillo $y$ fácil de obtener y coincide con otras comunicaciones que recomiendan emplearlo, especiabmente en países subdesarrollados, donde las personas tienen me. nos tejido adiposo, haciéndolo más representativo del perímetro muscular braquial ${ }^{15,16}$. Por el contrario el pliegue tricipital, mostró escasa asociación con las alteraciones de la relación $\mathrm{P} / \mathrm{E}$ y del perímetro braquial, lo cual sugiere deterioro mayor y más precoz de la masa magra y relativa conservación del tejido graso, que podría comprometerse más tarde y con mayor lentitud.

La albuminemia mostró tendencia a aumentar a medida que se prolongó la hospitalización, sugiriendo que su deterioro precoz pođría ser debido a disminución aguda e importante de la ingestión que se recuperaría rápidamente pasada la etapa inicial. En adultos no se han observado cambios en la albuminemia cuando se ha suprimido la ingestión de alimentos hasta por 3 semanas. En niños no se han realizado muchas observaciones al respecto, excepto el hecho clínico de no haber signos o síntomas de hipoalbuminemia. En nuestro estudio sólo 2 niños, con desnutrición pluricarencial evidente $\mathrm{e}$ hipoalbuminemia severa tuvieron manifestaciones clinicas. Si bien este parámetro puede no ser un buen indice del estado nutricional, habria que evaluarlo como factor pronóstico en pacientes en que permanece afectado, como ha sido sugerido por otros 17,18 .

$\mathrm{La}$ concentración de la hemoglobina estaba disminuida, desde el ingreso, en una proporción importante de pacientes, hecho que había sido consignado en un estudio previo realizado en Chile, en el que también se hacen notar diferencias significativas en la proporción de pacientes con concentraciones inferiores a las normales con respecto a la población en general. En nuestros pacientes es posible que esta deficiencia se asocie a factores etarios, socioeconómicos y de bajo peso al nacer, como también la severidad de las infecciones y la extracción de sangre para exámenes, pero su consideración excede los propósitos de nuestro estudio.

\section{RESUMEN}

Se estudiaron todos los lactantes entre 1 y 24 meses de edad hospitalizados por más de 10 dias entre Julio 1983 y Enero 1984 en el hospital Sótero del Río. Los pacientes fueron controlados semanalmente hasta el mes de estada, mediante peso, talla, perímetro braquial (PB), pliegue tricipital ( $\mathrm{PTr}$ ), albuminemia y $\mathrm{Hb}$. La hospitalización fue más prolongada cuando $\mathrm{P} / \mathrm{E}$ o $\mathrm{P} / \mathrm{T} \leqslant$ $90 \%$ al ingreso que en eutróficos $(18 \pm 9$ vs $31 \pm$ 28 días, $\mathrm{p}<0.05$ ); la diarrea fue más frecuente como causa de hospitalización en los pacientes con $\mathrm{P} / \mathrm{E} \leqslant 80 \%$. El $\mathrm{PB}<$ percentil 5 fue más frecuente en los lactantes que tuvieron hospitalizaciones más prolongadas y se ralacionó bien con $\mathrm{P} / \mathrm{E} \leqslant 80 \%$; el $\mathrm{PTt}<\mathrm{p5}$, no mostró mayores variaciones en los controles semanales. Se encontró baja prevalencia en hipoalbuminemia en controles iniciales $(<16 \%)$; y la proporción de pacientes con concentraciones deficientes de hemoglobina se mantuvo elevada entre los hospitalizados por tiempos prolongados $(>70 \%)$.

\section{REFERENCIAS}

1. Scrimshaw N.S.: Effect of infection on nutritional requirements. Am. J. Clin. Nutr. 30: 1536, 1977.

2. Mullen J.L., Kuzby G.P., Matheus D.C.: Reduction of operative morbidity and mortality by combined preoperative and postoperative nutritional support. Ann. Surg. 192: 604, 1980.

3. Cooper A., and Heird W.: Nutritional assessment of the pediatric patient including the low bittl weight infant. Am. J. Clin. Nutr. 35: 1132, 1982.

4. Trowbridge F.L., Staehling N.: Sensitivity and specificity of arm circunference indicators in- identifying malnourished children. Am. J. Clin. Nutr. 33: 687,1980 .

5. Mclaren D.S., Meguid M.M.: Nutritional assessment at the crossroads. J.P.E.N. 7: 575, 1983.

6. Miller C.L.: Immunological assays as measurements of nutritional status, a review. J.P.E.N. 2: 554, 1978.

7. Hamill P.V.V., Drizd T.A., Johnson C.L., Reed R.B., Roche A.F., Moore W.M.: Physical growth: Fational Center for Health Statistics percentiles. Am. J. Clin. Nutr. 32: 607, 1979.

8. Frisancho A.R.: Triceps skinfold and upper arm muscle size norms for assessment of nutritional status. Am. J. Clin. Nutr, 27: 1052, 1974.

9. Blackbourn G.L., Bistrian B.R., Maini R.J, Schkm H.J., Smith M.F.: Nutritional and metabolic assessment of the hospitalized patient. J.P.E.N. 1: 11,1977 .

10. Uouy R., Gazitíu R.: Evaluación del estado nutricional del enfermo hospitalizado. Rev. Méd. Cltil. 108: 542, 1980 .

11. Fuentes A.. Hertrampf E.. Barrera G. Heresi G., Jarpa $S$, Uouy $R$.: Evaluación nutricionaldel paciente pediátrico hospitalizado. Rev. Chil. Pediatr. 52: $387,1981$.

12. Gottás V., Fuentes A., Jarpa S., Uauy R.: Siłuación alimentatia de pacientes pediátricos hospitalizados. Rev. Chil. Pediatr. 52:397, 1981. 